\title{
Analysis of Vibratory Data Collected by the Space Acceleration Measurement System (SAMS) on Blue Origin, June 19, 2016
}

\author{
Kevin McPherson ${ }^{1}$, Eric Kelly ${ }^{2}$, Jennifer Keller ${ }^{2}$, Ajeeth Ibrahim $^{3}$, Erika Wagner ${ }^{4}$, and Kenneth Hrovat ${ }^{2}$ \\ ${ }^{I}$ NASA Glenn Research Center, Cleveland, OH; ${ }^{2}$ ZIN Technologies and NASA Glenn Research Center, \\ Cleveland, OH; ${ }^{3}$ NanoRacks, LLC, Webster, TX; ${ }^{4}$ Blue Origin, LLC, Kent, WA
}

\begin{abstract}
On Sunday, June 19, 2016, a Space Acceleration Measurement System triaxial sensor head flew on a suborbital flight aboard Blue Origin's New Shepard vehicle to collect precision vibratory accelerometry data. The Space Acceleration Measurement System (SAMS) sensor head was mounted inside of a Blue Origin single payload locker inside of the crew capsule. This paper describes the configuration, capture, and analysis of the SAMS data from this flight along with other, related flight log information provided by Blue Origin. Three overlapping periods during the flight were identified and characterized to provide future users of the platform with insight into options that may prove suitable for their research needs. Average
\end{abstract}

Key words Commercial Spaceflight; Suborbital; Microgravity Vibratory Environment; Acceleration Measurement; Platform Characterization; Vibratory Data Analysis; Microgravity Research; Pathfinder

Correspondence to: Kenneth Hrovat NASA Glenn Research Center 21000 Brookpark Road Mail Stop 333-1

Cleveland, OH 44135

Telephone: 216-433-5376

E-mail: hrovatk@zin-tech.com accelerations in the Post-Separation Period were consistent with other low- $g$ research platforms, while the shorter Microgravity Period in the middle of the flight showed ultra-quiet vibratory acceleration environments. Researchers can consider this microgravity quality versus time a tradeoff in their experimental designs.

\section{INTRODUCTION}

Blue Origin's New Shepard is a suborbital reusable launch vehicle. After liftoff, the vehicle ascends vertically for approximately $150 \mathrm{~s}$ before main engine cutoff in the upper atmosphere. Several seconds after engine cutoff, the capsule separates from the booster and is pushed away by mechanical springs. From this point, the capsule and booster coast and reenter separately. The vehicles experience microgravity for a period of about 3 min before returning to Earth. The booster maneuvers back to a landing pad, restarts its engine, and deploys landing gear to perform a rocket-powered vertical landing. The capsule reenters and lands under a three-parachute canopy with the assistance of a retro-thrust system to reduce landing loads. The entire flight typically occurs autonomously, without flight or ground crew intervention.

On January 11, 2016, the Space Acceleration Measurement System (SAMS) team was approached by NASA Headquarters (HQ) regarding flying an accelerometry unit on an 
upcoming flight on Blue Origin's New Shepard vehicle. Given the flight history of the Space Acceleration Measurement System as a microgravity measurement system, NASA HQ sought to use SAMS to understand the microgravity vibratory environment available on this suborbital vehicle.

The SAMS is primarily used for precision accelerometry aboard the International Space Station (ISS). This system was designed to characterize the vibratory regime, and therefore cannot measure accurately low-frequency, lowmagnitude (residual) acceleration. Multiple triaxial sensor heads located throughout the ISS send acceleration measurements to a centralized control unit, which is used for configuration control, commanding, and data downlink. These data are continuously streamed to the ground. The Blue Origin flight configuration used the same SAMS sensor head without a centralized control unit, allowing for the relatively short turn-around needed to meet mission readiness milestones.

Logistically, the Blue Origin flight most closely resembled previous SAMS missions on parabolic aircraft, sounding rockets, and drop towers dating back to 1991. This flight on Blue Origin's New Shepard was the second time the SAMS had been used on a commercial vehicle,

A

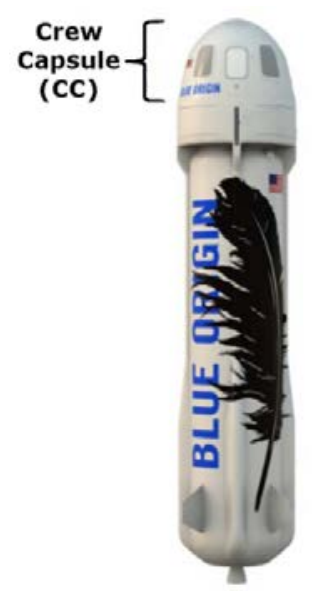

B



the first being its use on the Zero-G aircraft in 2008.

\section{MATERIALS AND METHODS}

\section{Acceleration Measurement System Description}

The SAMS unit was launched within a Blue Origin Single Payload Locker mounted on a stack inside the pressurized capsule cabin (Figure 1). Blue Origin's flight accelerometers, which were used for correlation, were similarly hard mounted inside the cabin. The SAMS X-axis was aligned with the vertical flight direction, pointing towards the nose of the capsule.

The mechanical interface between the SAMS and Blue Origin's New Shepard vehicle was simply the sensor head bolted inside a Single Payload Locker. The electrical interface consisted of pigtails through two large Single Payload Locker connectors, which were provided by Blue Origin. This supplied SAMS with the power and data connections needed to operate within the prescribed architecture. A DC-DC converter was used as an adjunct to a custom cable that transmitted power, Ethernet, and serial communications between the sensor and the Blue Origin Payload Controller, where measurement data were recorded.

C

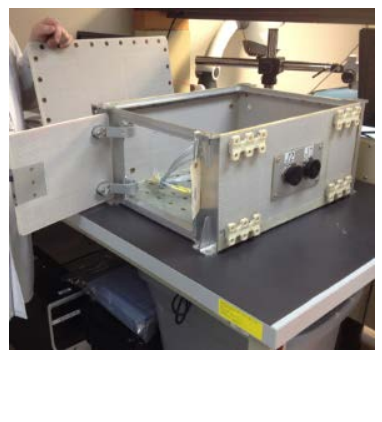

D

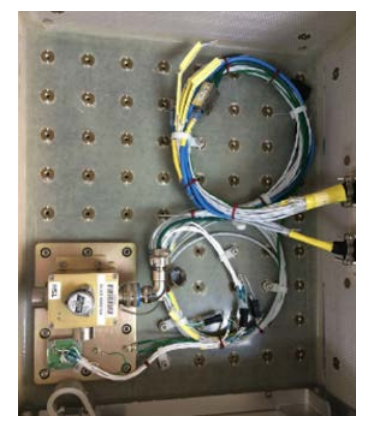

Figure 1. SAMS sensor location within the New Shepard vehicle. (A) The New Shepard vehicle, showing the capsule on top. (B) A top-down cross-sectional view of the capsule with six payload stacks uniformly distributed around its perimeter; the payload locker that housed SAMS was at about the 8 o'clock, position \#3. (C) A perspective view of the SAMS payload locker. (D) A top-down view of the SAMS sensor mounted within its payload locker with cabling for power and data. 
The SAMS Triaxial Sensor Head-Ethernet Standalone (TSH-ES S/N 14) used on New Shepard was configured to take acceleration measurements without requiring a control unit. The sensor head collected measurements simultaneously on three orthogonal axes at a rate of $500 \mathrm{samples} / \mathrm{s}$. Each of the three orthogonal sensors had a practical pass-band from 0.01 to 204.2 Hz. Unity gain was used, resulting in large magnitude accelerations being clipped, per-axis, at about $1.84 \mathrm{~g}$. This gain setting provided maximum resolution during free fall.

The sensor was powered on during a prelaunch, automated sequence. After booting up, it could obtain measurements continuously, limited only by battery and recording capacity. Total recording time for this flight was $15.7 \mathrm{~min}$. Recording spanned continuously from $163 \mathrm{~s}$ prelaunch to $169 \mathrm{~s}$ post-landing.

\section{Data Processing and Analysis}

To correlate SAMS acceleration data with flight events recorded by Blue Origin over the course of the flight, it was necessary to first perform post-flight processing with interpolation and cross-correlation between the SAMS acceleration data and the Blue Origin flight log information. These calculations showed that the SAMS acceleration data led Blue Origin by $163.255 \mathrm{~s}$, ignoring structural transmission and propagation time between the location of the Blue Origin accelerometer and the SAMS sensor.

The remainder of this document will focus on describing the results obtained from considering three overlapping periods surrounding the flight's apogee, in order to provide future users of Blue Origin's New Shepard platform with general characterization that may prove suitable to their research needs. Note that the mean value computed during the Microgravity Period is subtracted from the SAMS data in these three periods. This pre-processing step removes the sensor bias and puts all three periods on a common baseline relative to the Microgravity Period. Additionally, the power spectral density (PSD) was computed using Welch's averaged, modified periodogram method and the cumulative root mean square (RMS) versus frequency was computed using Parseval's theorem (Oppenheim and Schafer, 1975).

\section{RESULTS}

There can be widely differing objectives and requirements with regard to the platform on which a given payload's near-weightless investigations should be conducted. This paper is not intended as a comprehensive analysis to cover all of those conditions. Instead, we aim here to present a clear and incisive accounting of some of the key features and characteristics of the vibratory environment as measured by the SAMS on Blue Origin's New Shepard vehicle. We do so by describing three different periods during the flight as measured by the SAMS sensor in a representative payload locker location. The most intuitive period to describe is the Coast Period, between capsule stabilization and the start of reentry. This phase is quite well defined and delimited by the Blue Origin flight log's Coast Start and Coast End events, providing researchers with convenient trigger points. On the other hand, the Post-Separation Period and the microgravity period were defined more pragmatically on the basis of the flight data. A synopsis of all three periods is shown with three subplots of the SAMS sensor X-, Y-, and Z-axis measurements in Figure 2.

For a spectral overview of the vibratory environment during the New Shepard flight on June 19, 2016, we computed and plotted a spectrogram (Figure 3). This three-dimensional plot helps to show boundaries, patterns, and structure in time along the horizontal axis and frequency along the vertical axis. The third dimension, represented by color, only crudely shows magnitudes in the form of color-mapped PSD values. This spectrogram combines the three independent SAMS measurement axes via rootsum-squares of the per-axis PSDs.

\section{Post-Separation Period}

The Post-Separation Period, spanning from 1 $\mathrm{s}$ after the flight log's Separation event to the Coast End event, lasted approximately $2.8 \mathrm{~min}$. This period provides the longest duration for a quiescent vibratory environment; however, multiple, small impulsive accelerations occurred during this period as expected. These impulses were the result of reaction control thruster firings immediately after separation and upon atmospheric reentry to control vehicle orientation. 
SAMS TSH-ES S/N 14, Payload Locker Location, 204.2 Hz

Post-Separation Period

19-June-2016
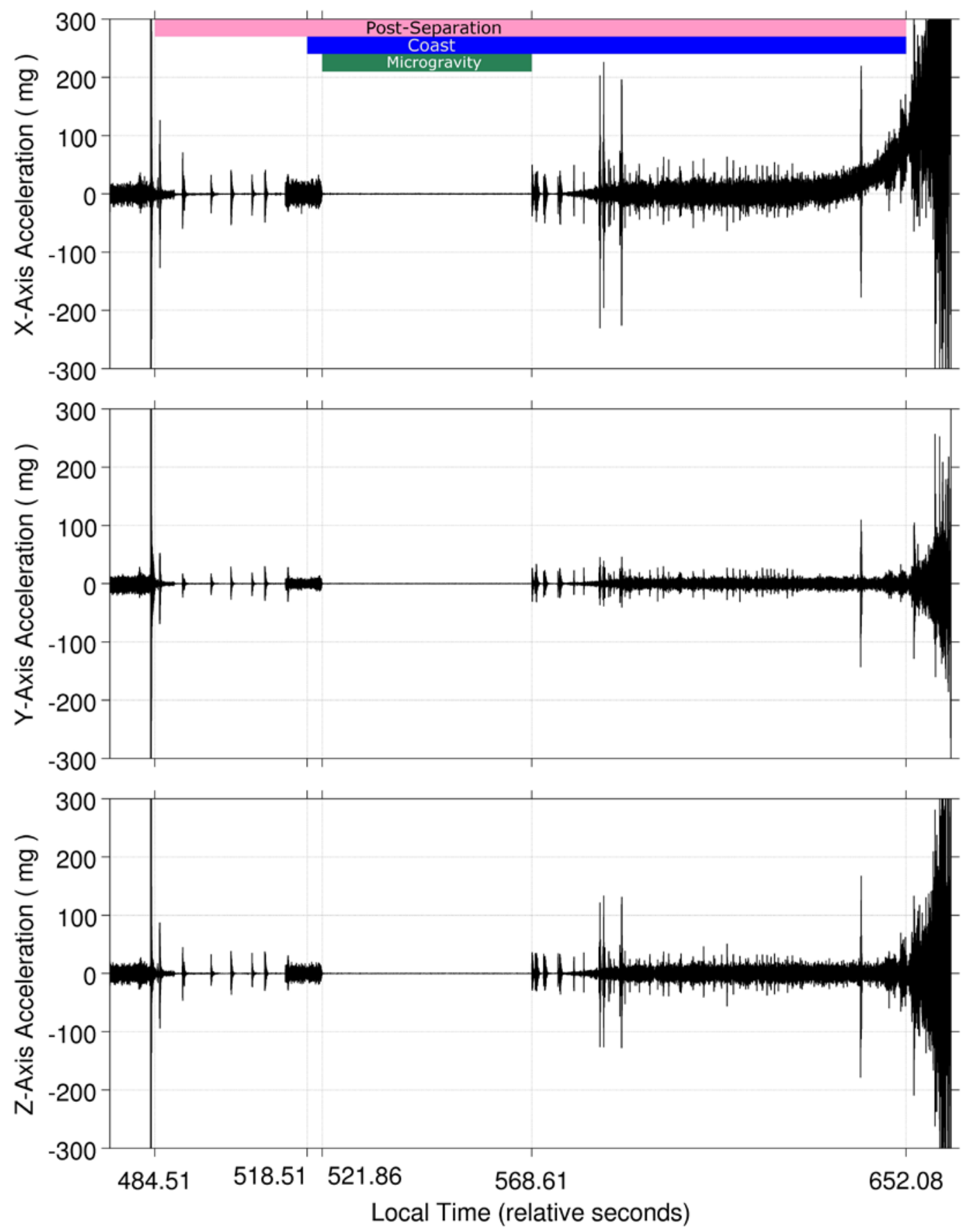

Figure 2. Per-axis plot of SAMS acceleration measurements up to $204.2 \mathrm{~Hz}$ versus time for three main periods. Note that the mean value computed during the Microgravity Period is subtracted off. The colored bands at the top represent flight periods. The pink band (top) demarks the Separation Period, which was $\sim 2.8$ min in duration from $1 \mathrm{~s}$ after the Separation event to the Coast End event. The blue band (middle) indicates the Coast Period, which was $\sim 2.2 \mathrm{~min}$ in duration from the Coast Start event to the Coast End event. The green band (bottom) marks the very quiet Microgravity Period, which was $\sim 46.8 \mathrm{~s}$ in duration from $521.86 \mathrm{~s}$ to $568.61 \mathrm{~s}$. 


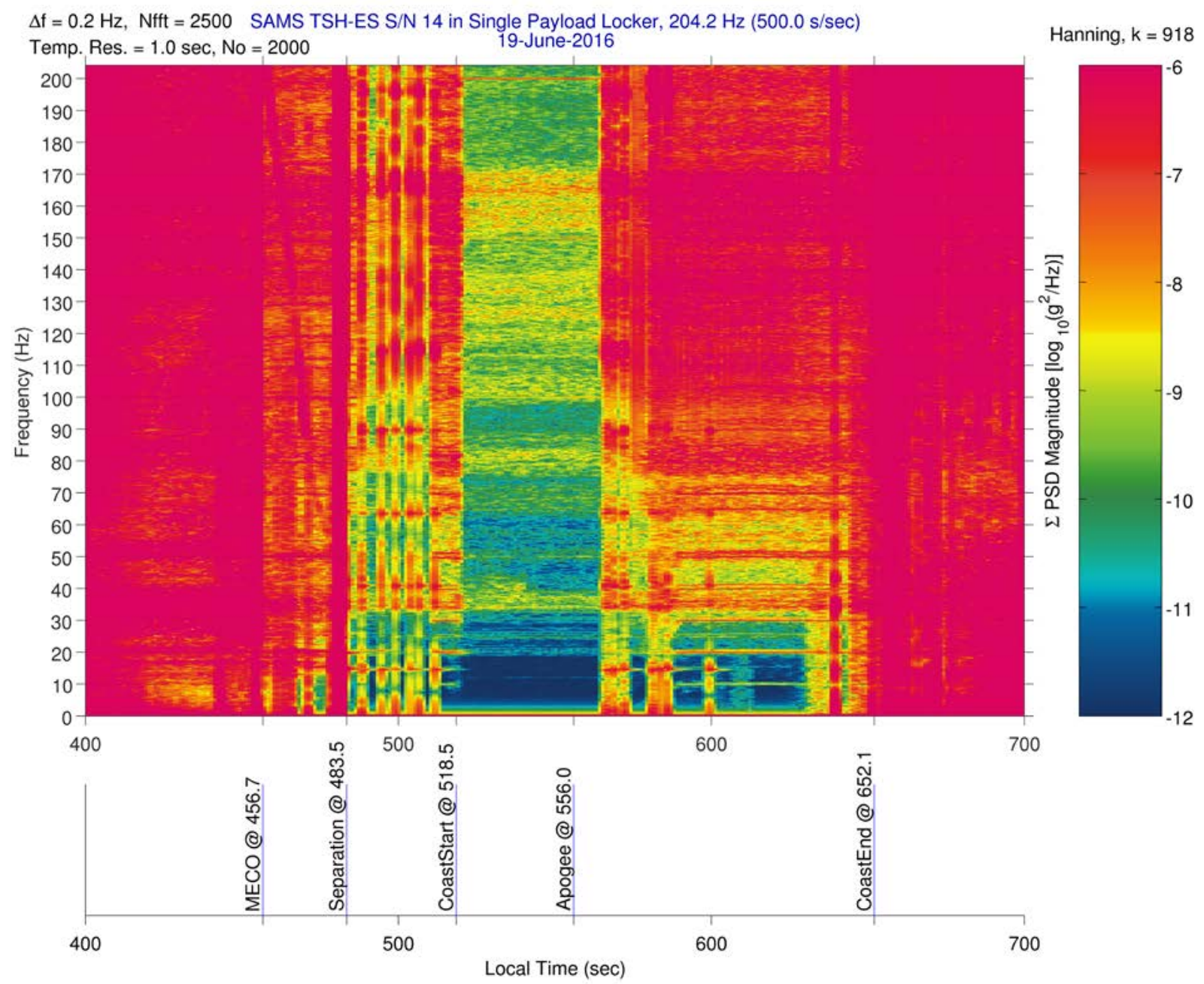

Figure 3. Spectrogram computed from SAMS measurements and Blue Origin flight information. This threedimensional plot shows the acceleration spectrum via color-mapped power spectral density (PSD) magnitude versus frequency on the vertical axis, and versus time on the horizontal axis. The PSD color scale was chosen for emphasis during free fall and represents the squared deviation of the acceleration with respect to the mean acceleration during free fall. Although a Hanning window was used to reduce spectral leakage for each of the constituent PSDs in this spectrogram $(k=918)$, the strong signal near zero frequency should be disregarded as attributable to spectral leakage from sensor bias.

Table 1 shows the RMS acceleration levels for two different frequency limits that were calculated for the Post-Separation Period, where the total value was calculated from the root sum of squares of the per-axis PSDs. Table 2 shows the five- number statistical summary of acceleration magnitudes that was also computed (Table 2), where the total value is that of the vector sum of the per-axis components.

Table 1. Root-mean-square accelerations calculated for the Post-Separation Period.

\begin{tabular}{|l|c|c|c|c|}
\cline { 2 - 5 } \multicolumn{1}{c|}{} & \multicolumn{4}{c|}{ Root-Mean-Square Acceleration (mg) } \\
\hline Frequency & X-Axis & Y-Axis & Z-Axis & Total \\
\hline Below $50 \mathrm{~Hz}$ & 0.25 & 0.94 & 0.75 & 1.23 \\
\hline Below $200 \mathrm{~Hz}$ & 8.60 & 3.66 & 6.06 & 11.14 \\
\hline
\end{tabular}


Table 2. Five-number statistical summary of acceleration magnitudes during the Post-Separation Period.

\begin{tabular}{|c|c|c|c|c|c|}
\hline \multirow[b]{2}{*}{ Percentile } & \multicolumn{4}{|c|}{ Acceleration Magnitude (mg) } & \\
\hline & X-Axis & Y-Axis & Z-Axis & Total & \\
\hline 25 th & 0.28 & 0.17 & 0.20 & 0.47 & \\
\hline 50 th & 1.45 & 0.62 & 0.92 & 3.37 & Median \\
\hline 75th & 8.60 & 3.07 & 5.01 & 11.91 & \\
\hline 97.5 th & 51.11 & 10.25 & 16.92 & 54.75 & \\
\hline MAX & 230.48 & 143.29 & 178.41 & 276.18 & \\
\hline
\end{tabular}

\section{Coast Period}

The Coast Period, spanning from the flight log's Coast Start event to the Coast End event, was approximately $2.2 \mathrm{~min}$ in duration. This period, a subset of the Post-Separation Period, was well-defined in terms of flight log event markers. This provided a relatively long duration for a quiescent vibratory environment; however, impulsive accelerations due to thruster firings were prominent here as well, due to reaction control system (RCS) firings to compensate for unexpected vehicle dynamics near the end of the Coast Period.

The RMS acceleration levels are shown in Table 3, and Table 4 shows the five-number statistical summary of acceleration magnitudes, which were computed for the Coast Period as they were for the Post-Separation Period above.

\section{Microgravity Period}

The Microgravity Period was partitioned as the longest contiguous span when the SAMS acceleration vector magnitude was less than $4 \mathrm{mg}$. This was an empirical selection based on an overtly quiet time range that was a subset of the Coast Period. The Microgravity Period lasted 46.8 $\mathrm{s}$ and was devoid of any thruster firings. This provided a significantly quieter vibratory acceleration environment. Tables 5 and 6 show the RMS acceleration levels and five-number statistical summary of acceleration magnitudes, computed as described above.

Table 3. Root-mean-square accelerations calculated for the Coast Period.

\begin{tabular}{|l|c|c|c|c|}
\cline { 2 - 5 } \multicolumn{1}{c|}{} & \multicolumn{4}{c|}{ Root-Mean-Square Acceleration (mg) } \\
\hline Frequency & X-Axis & Y-Axis & Z-Axis & Total \\
\hline Below $50 \mathrm{~Hz}$ & 0.32 & 1.23 & 1.00 & 1.23 \\
\hline Below $200 \mathrm{~Hz}$ & 10.10 & 4.13 & 7.03 & 11.14 \\
\hline
\end{tabular}

Table 4. Five-number statistical summary of acceleration magnitudes during the Coast Period.

\begin{tabular}{|c|c|c|c|c|c|}
\hline \multirow[b]{2}{*}{ Percentile } & \multicolumn{4}{|c|}{ Acceleration Magnitude (mg) } & \\
\hline & X-Axis & Y-Axis & Z-Axis & Total & \\
\hline 25 th & 0.29 & 0.18 & 0.21 & 0.47 & \\
\hline 50 th & 2.38 & 0.91 & 1.46 & 5.13 & Median \\
\hline 75 th & 10.29 & 3.51 & 5.81 & 13.68 & \\
\hline 97.5 th & 57.34 & 10.56 & 17.64 & 59.93 & \\
\hline MAX & 230.48 & 143.29 & 178.41 & 276.18 & \\
\hline
\end{tabular}


Table 5. Root-mean-square accelerations calculated for the Microgravity Period.

\begin{tabular}{|l|c|c|c|c|}
\cline { 2 - 5 } \multicolumn{1}{c|}{} & \multicolumn{4}{c|}{ Root-Mean-Square Acceleration (mg) } \\
\hline Frequency & X-Axis & Y-Axis & Z-Axis & Total \\
\hline Below $50 \mathrm{~Hz}$ & 0.01 & 0.07 & 0.03 & 0.08 \\
\hline Below $200 \mathrm{~Hz}$ & 0.32 & 0.20 & 0.23 & 0.45 \\
\hline
\end{tabular}

Table 6. Five-number statistical summary of acceleration magnitudes during the Microgravity Period.

\begin{tabular}{|c|c|c|c|c|c|}
\hline \multirow[b]{2}{*}{ Percentile } & \multicolumn{4}{|c|}{ Acceleration Magnitude (mg) } & \\
\hline & X-Axis & Y-Axis & Z-Axis & Total & \\
\hline 25 th & 0.10 & 0.06 & 0.07 & 0.25 & \\
\hline 50 th & 0.22 & 0.14 & 0.16 & 0.36 & Median \\
\hline 75 th & 0.37 & 0.24 & 0.27 & 0.52 & \\
\hline 97.5th & 0.76 & 0.48 & 0.56 & 0.94 & \\
\hline MAX & 2.27 & 1.18 & 1.45 & 2.77 & \\
\hline
\end{tabular}

\section{DISCUSSION}

We focused on three main periods most relevant to low- $g$ researchers during this flight of New Shepard to give users an array of results showing a tradeoff between, primarily, the duration of quiescence and acceleration magnitude levels. Notably, the period of free fall was reduced on this flight compared to other launches of the system because of RCS firings to compensate for vehicle dynamics. The root cause has been identified and addressed, and future flight designs are expected to increase the duration of quietest flight and improve environments throughout free fall. Per the New Shepard Payload User's Guide (Blue Origin, 2017), on a nominal mission, nearly all RCS thruster firings will happen during the 10 to $15 \mathrm{~s}$ immediately following separation as the capsule nulls rates in pitch, yaw, and roll axes induced by any asymmetries in the mechanical separation spring force or residual aerodynamic disturbances. After this period, the frequency of RCS thruster firings is expected to drop significantly. After the start of the Coast Phase, approximately $35 \mathrm{~s}$ after separation, the RCS thrusters are not expected to fire again until reentry, when the sensed acceleration due to aerodynamic drag will be far greater than any RCS-induced acceleration.
A network interface and software supplied to Blue Origin Payload Controller users allows for real-time monitoring of vehicle mission data such as the Coast Start/End events mentioned earlier. This information can be used to trigger scripted actions based on these data. This can be leveraged by payloads to control various aspects of their operations using these triggered actions.

The longest quiescent period observed was the Post-Separation Period, which on this flight lasted about $2.8 \mathrm{~min}$. The start of this period was selected to be $1 \mathrm{~s}$ after separation to allow recoil/ringing to damp out. At the reentry end of the analysis window, an alternative end point for this period might be when the $\mathrm{X}$-axis acceleration (perhaps a sliding mean value) starts diverging from the Microgravity Period's mean value. Researchers interested in ultra-quiet environments to support their science goals should consider isolating payloads against vibrations greater than $50 \mathrm{~Hz}$ to significantly reduce $g$-jitter across the Post-Separation Period. SAMS measurements on this flight suggest an attenuation factor approaching an order of magnitude when using 50 $\mathrm{Hz}$ vibration isolation. Low-cost, passive isolation measures such as rubber grommets can provide attenuation at the higher-frequency portion of the vibratory acceleration environment, although, this will come at the cost of low-frequency 
amplification as passive isolators move vibration energy from higher frequencies to lower frequencies. Structural modeling or vibration lab testing could then be used to further hone the specifications for passive isolation hardware.

The Coast Period was similar in character and duration to the Post-Separation Period, with the only potential advantage for researchers being its better-defined Coast Start and Coast End event markers available for triggering at the cost of a somewhat shorter duration.

While the Microgravity Period was only 46.8 $\mathrm{s}$, the vibratory acceleration environment in this span provides a much quieter vibratory environment, with acceleration magnitudes on all three measurement axes roughly an order of magnitude smaller. Blue Origin also predicts the duration of this period will improve in future flights.

When we consider a widening bandwidth in the form of a cumulative RMS acceleration versus frequency plot, we can potentially address various investigators' concerns across the entire bandwidth measured by the SAMS sensor (Figure 4). When this is plotted, we see that the PostSeparation Period closely resembles the Coast Period, albeit for a longer duration. Furthermore, we see that the Microgravity Period stays well below $1 \mathrm{mg}$ up to the sensor cutoff frequency of approximately $200 \mathrm{~Hz}$. Comparing

RMS acceleration levels below $10 \mathrm{~Hz}$, see Figure 5, shows that the best New Shepard vibratory environment is superior to the vibratory environments of the complete test periods of parabolic flight, traditional sounding rockets, ISS, and even drop towers. Note too that the entire Coast Period in New Shepard is shown to be comparable to that of traditional more expensive single-use sounding rockets. Further study of aerodynamic drag data would round out characterization of the micro- $g$ environment on New Shepard and other similar vehicles.



Figure 4. A cumulative RMS acceleration versus frequency plot for the three main periods of interest. The Post-Separation Period (red) and Coast Period (blue) mostly track each other, and the New Shepard Microgravity Period stays well below $1 \mathrm{mg}$ RMS up to the sensor cutoff frequency. 


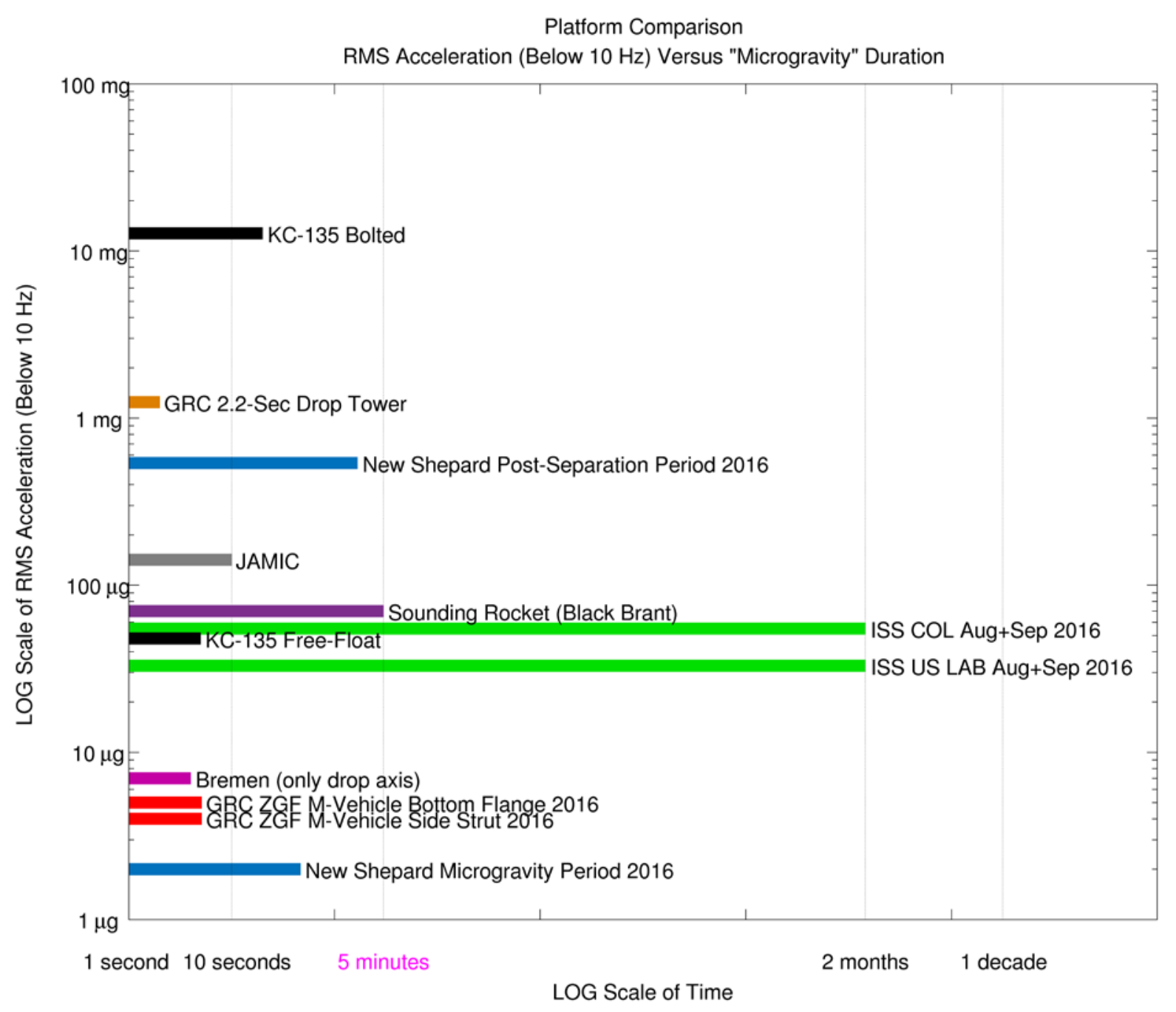

Figure 5. Comparison of RMS acceleration levels below $10 \mathrm{~Hz}$ versus microgravity duration for several different types of platforms (Ross, 2001).

\section{ACKNOWLEDGEMENTS}

This work was supported by the NASA SpaceDOC II contract (NNC14CA02C). The authors would like to acknowledge ZIN Technologies for their expertise and resourcefulness during an abbreviated schedule to meet mission deadlines. A special thanks to Bill Pachinger for his help getting the SAMS custom cabling built, tested, and ready for flight. Also, thank you to Brad Humphreys at ZIN Technologies for his expertise, feedback, and goto knowledge of structural dynamics.

We would also like to thank the Blue Origin team for their hospitality and resources provided during payload checkout and integration at the launch site. This work was supported by NASA HQ under the guidance of Diane Malarik and Angel Otero.

\section{REFERENCES}

Blue Origin (2017) New Shepard Payload User's Guide For Research and Education Missions. NSPM-MA0002-C Rev C. Available via request at https://www.blueorigin.com/

Oppenheim AV, Schafer RW (1975) Digital Signal Processing. Englewood Cliffs, NJ: Prentice-Hall

Ross HD (2001) Microgravity Combustion: Fire in Free Fall. San Diego, CA: Academic Press 